\title{
Color Experience: A Semantic Theory ${ }^{*}$
}

\section{Mohan Matthen}

The following is a semantic theory of color experience.

Color experience represents or denotes color properties, and attributes these properties to visual objects.

On such a theory, color experience informs us about the external world by means of a semantic relationship, representation or denotation, that it bears to external-world properties, and it attributes these properties to external objects (that it also denotes, though this is not something that I will take up in what follows).

The use of concepts such as representation is essential to any semantic theory. In the specific version of the theory that I offer,

(a) the above semantic relationships operate through the vehicle of a systematic set of similarity relations.

I shall argue that

(b) our grasp of these perceptual relations is (for the most part) innate.

I shall also argue

(c) that the similarity relations in question are dynamic, in the sense that they are constitutively linked to certain cognitive changes in the perceiver subsequent to color perception.

\footnotetext{
* Many thanks to Stephen Biggs, Jonathan Cohen, and Reinhard Niedereé for detailed
} comments, each of which influenced the content of this paper. 
It is a central feature of my presentation that it relies on distinctions concerning the degree of certainty that attaches to different kinds of proposition that we come to know through color perception.

\section{The Evidential Status of Color Vision}

Consider a statistically normal human colour perceiver, Trich (so-called for her trichromacy) viewing a piece of fruit in reasonably good light. Suppose that to Trich this fruit looks orange. Trich's visual experience of the fruit, $O$, is evidential in a number of ways.

A. Object-Knowledge Trich can take $O$ as strong support for the proposition:

OK (1) That [fruit] is orange.

If Trich is in any doubt about (1), she could improve her viewing conditions: she might put on her glasses; she might take the fruit to the window, where the sunlight is stronger; she might try to eliminate any sources of non-standard illumination; she might turn the fruit over in her hands or bring it closer to her eyes. At the end of this process, she would be in a state of empirical certainty about (1). By empirical certainty, I mean this: Trich has no isolated reason for doubting (1) - no reason that would not simultaneously lead her to doubt a whole raft of unconnected propositions.

Empirical certainty does not entitle her to dismiss sceptical doubt. She may be dreaming, deluded, or deceived by an evil demon; she may be a brain in a vat; her visual pathways may be subject to a strange form of transcranial interference. This kind of doubt is not, however, isolated. Since sceptical doubt attacks background conditions for empirical knowledge, it attacks not only (1), but equally many other propositions. The possiblilities just mentioned would lead her in turn to doubt whether the lemon next to the orange was really yellow, whether it was really her hand in which the fruit resided, whether it was really daylight, whether she was really awake, and so on. Sceptical doubt spreads by undermining the foundations of empirical knowledge. Empirical certainty is subject to the security of these background conditions. 
My point is that given careful visual inspection, Trich may still doubt (1), but only in an expansive way.

B. Color-Knowledge In light of her experience, $O$, Trich is also entitled to certainty regarding certain propositions about the color presented therein. To wit:

CK (2) The color presented in $O$ is yellowish.

CK (3) The color presented in $O$ is less reddish than that presented in another visual state $C$ (which happens to be occasioned by a cherry).

CK (4) The color presented in $O$ is less yellowish than that presented in another visual state $L$ (which happens to be occasioned by a lemon).

CK (5) The color presented in $O$ is more similar to that presented in $L$ than it is to that presented in $C$.

These propositions concern colors, not the objects that possess them.

Now, Trich's certainty with regard to CK propositions is actually higher than empirical certainty. For, it seems that to know the above sort of fact about the colours, one needs only to experience them (cf. Johnston 1992, Hellie 2005). For Trich to know that the color of the thing she seems to see is yellowish, as asserted in (2), she needs nothing more than her experience of that color. She does not need to know that the fruit actually is the colour it seems to be; she does not even need to know that the fruit exists. Even if Trich's experience, $O$, is a dream or a hallucination, it is an experience of a certain color, and this is enough for her to be sure that the color is yellowish in hue. Hallucinatory color-experiences are no less probative with regard to the colorknowledge of the sort contained in (2) - (5) than normal veridical experiences. For this reason, Trich is entitled to dismiss even sceptical doubt with respect to the CK propositions. She is entitled to Cartesian certainty with regard to them. Cartesian certainty is certainty that is proof even against sceptical doubt. 


\section{Color Experience}

How can Trich be entitled to so strong a level of certainty regarding the colors presented in her color experiences? You might think that one could possess such certainty only with respect to subjective facts - Cartesian certainty starts with that which can be introspected. (Famously, Descartes argued that it attaches to the proposition "I am", and to the content of ideas that are clearly and distinctly apprehended, such as that matter is spatially extended, but consciousness is not.) However, color-attribution is objective - whether or not the fruit Trich is looking at is orange does not depend just on her or her state of mind. Given that this is so, orange cannot be merely a subjective or private category. It isn't like "This fruit gives me pleasure" - of which she can be certain because it is true in virtue of an subjective state that she can introspect. How can she be so certain of something that she doesn't determine in the same way?

I shall argue that the entitlement arises out of the fact that colour-knowledge propositions (2) - (5) express facts arise out of Trich's (largely) innate representational scheme for colors. These propositions are about colors that exist independently of Trich, but her knowledge of the similarity relations among these colors and of their compositional structure arise out of this representational scheme.

C. Unexperienced Colors Trich is able visually to image a "missing shade" of yellowy orange "in between" the color presented by this fruit and that presented by the lemon, even though she happens never actually to have encountered anything of such color (cf. Hume's missing shade of blue, Treatise §1). She is not dependent on memory to know of such a color. Moreover, she can be induced to have experiences of "impossible" colors: colors that are both reddish and greenish (Crane and Piantanida 1983) and colors that lie outside the color solid (Churchland, this volume). In virtue of such experiences, she apprehends propositions analogous to the CK propositions above with regard to colors that can never be instantiated in real objects. She is entitled to Cartesian certainty with regard to these colors too, even though she never sees them 


\section{Color Experience}

"for real". Having imagined them, she is entitled to be certain of whether they are bluish or yellowish, bright or light, and so on.

It is perhaps worth emphasizing that unexperienced colors are not just idle artefacts of the philosophical museum. It is easy to imagine a rainy equatorial forest in which blue and saturated yellow is never visible, or some particularly sheltered environment in which blood is never shed, and men never see red. But even putting such fantasies aside, the dye-deprived cultures of antiquity did not have access to all the colours. Yet, even such chromatically deprived people were capable of "raising up" (as Hume put it) the ideas of the missing shades. And when they did, they would have been entitled to Cartesian certainty regarding facts analogous to those expressed by (2) - (5) above.

Knowledge about colours that one has never encountered has to be a priori. How can one have a priori certainty about things that exist independently of oneself?

D. Object Appearance Knowledge Finally, in light of her experience $O$ - and $O$ alone Trich also possesses Cartesian certainty about one other proposition:

OAK (6) That [fruit] looks orange.

OAK propositions, like OK propositions, are about the fruit. They are evidence for object-knowledge, but not strong enough evidence to override sceptical doubt regarding knowledge about objects.

(6) is trivial on my description of $O$ - for I stipulated at the outset that $O$ is the experience of the fruit looking orange. This triviality does not lessen the interest of the observation. Undergoing an experience of the O-type - however that experience is to be described - gives Trich Cartesian certainty concerning (6). How can that be? This is related to the CK propositions, $(2)-(5)$. What does it say about the colors that we are entitled to certainty concerning certain facts about them? And with regard to (6): what is it about our knowledge of orange that we are entitled to be certain that the fruit looks orange. Compare: "That fruit looks like a lemon". This demands not only that one 
knows what that fruit looks like, but also what a lemon looks like. Why is something like this not true of $(6)$ ?

In order to be certain that something looks orange, one needs to be certain about relevant facts concerning the nature of the colour, orange. How does one come to be so? Since one cannot have Cartesian certainty of what objects look like even after viewing them, the source of one's certainty regarding the color orange must be different.

\section{Secondary Quality Theories of Color}

Some theories make it a part of the essence of color that they produce a certain kind of sensation - "secondary quality" or "response-dependence" theories, as they are called. How would they account for the evidentiary status of color vision as outlined in the previous section?

A. According to Dispositionalism, color is a disposition in things to evoke in "normal" perceivers a certain type of sensation in "normal" circumstances. According to this theory, a fruit is orange in virtue of the fact that it possesses a disposition to create a certain type of color sensation, of which $O$ is an example. Dispositionalists suppose that the Cartesian certainty of Trich's color-knowledge arises from the fact that she has incorrigible access to her own sensations, which have the properties in question reddishness, yellowishness, etc. Facts concerning the compositionality of color as in (2) - (4) and similarity relations as in (5) ride on some quality of the sensation, according to the Dispositionalist, and are thus knowable with certainty.

These theorists also have a partial account of Trich's merely empirical certainty with regard to object-knowledge. When she first looks at the fruit, Dispositionalists say, she is certain only of the sensation that the fruit evokes in herself in her particular circumstances of viewing. This is some evidence that it possesses a disposition to evoke this sensation in normal circumstances, but not conclusive evidence. By examining it closely in a variety of normal viewing conditions - by bringing it to the window, turning 
it over, and putting on her glasses - she expands the range of circumstances in which she has viewed the fruit. Thus, she increases her certainty about its dispositions with respect to her own sensations in normal circumstances of viewing. However, such certainty is subject to sceptical doubt - this kind of scrutiny does not rule out the possibility that she is systematically deluded about her circumstances.

At first sight, then, Dispositionalists can account for the distinctive kinds of certainty possessed by the OK and CK propositions. There is, however, an obstacle on the dialectical path to object knowledge - Trich's empirical certainty regarding the color of something she sees. According to Dispositionalists, the color of the fruit is not perceiver-dependent; it is in the fruit. Its color is its disposition not only to create a certain kind of sensation in Trich, but in other normal perceivers as well. Looking at the fruit in all kinds of different circumstances certainly helps Trich know its propensities with regard to her own color experiences - but it does not help her know how it is disposed with regard to other perceivers. Examining the fruit closely could not help her much with this; this procedure only gives her broader knowledge of the fruit's dispositions to evoke sensations in herself. (In fact, as we shall see in section VIII, it is most likely false that things elicit exactly the same sensations in different perceivers.) To draw any conclusion about what sensations it creates in other perceivers requires other arguments - including, perhaps, an argument by analogy. No such argument is compelling enough to provide Trich with anything like empirical certainty.

So the Dispositionalist cannot, in the end, account for the intuition I advanced in section I.A above that Trich can arrive at empirical certainty about object-knowledge.

A related problem arises for Cartesian certainty of knowledge concerning the apparent color of the fruit. Given her definition of 'orange', the Dispositionalist glosses OAK (6) as follows: 
(D6) That fruit looks as if it possesses a disposition to create an orange sensation in normal perceivers in normal circumstances. ${ }^{1}$

We have just argued that Trich's experience fails to give her empirical certainty about the experiences of others. (D6) falls short of empirical certainty for exactly the same reason, and so it falls short of Cartesian certainty as well.

On the Dispositionalist's account, object-appearance knowledge does not come out more certain than object-knowledge - rather, it possesses exactly the same credibility. Moreover, both object-knowledge and object-appearance knowledge regarding color fall short of empirical certainty. This is surely a problem.

B. The problem with Dispositionalism is that it posits too great an evidential gap between sensation and color as they understand color. Relationalist accounts of color such as those of Brian McLaughlin (2003) and Jonathan Cohen (2004, this volume, forthcoming) reduce this evidential gap to almost nothing. Consequently, they do better on these tests. But their position on the evidential relationship between object appearance knowledge and object knowledge is quite implausible.

The same thing may look different colors in different conditions of viewing. When something looks white in white light and orange under a sodium bulb, is it really white or really orange? According to McLaughlin and Cohen, both options give undue precedence to one set of circumstances over the other. Relationalism is their way of unravelling this Gordian knot: on this position, orange is a many-place predicate that has as many parameters as the determinants of colour experience. Thus, things are not orange as such, or white as such, but (simplifying somewhat) orange relative to an observer, given certain circumstances of viewing, and white relative to another observer, or relative to different circumstances of viewing. Thus, orange is a relation, not a simple unary predicate as it appears in ordinary language and thought. To say that

1 (D6) is the Dispositionalist's gloss on (6), above. I'll use this kind of notation in what follows. 
a fruit is orange is syntactically incomplete in exactly the same way as it is syntactically incomplete to say that New York is in between. (In between what and what? Orange relative to whom and what circumstances?) When we say that something is orange tout court, we assume some way of filling in the extra parameters. For example, we might mean that it is orange for observer $O$ (or some set of observers) in circumstances of viewing $C$ (or some range of circumstances).

At first sight, Relationalism runs into an immediate difficulty. For Relationalists, the most specific way to take object-knowledge (1) in the circumstances we have been considering is this:

(R1) That fruit is orange for Trich in the circumstances of viewing that obtained when she viewed it.

To determine the truth of this proposition, Trich needs nothing but her own experience. Her certainty should thus be Cartesian (too high by my reckoning). And of course, exactly the same is true of (6), which concerns the color-look of the fruit. So the Relationalist cannot account for the difference between (1) and (6).

McLaughlin and Cohen have another option, however, with respect to the fruit's looking orange. They could introduce normalcy in some such way as the following:

(R1') That fruit is orange to normal perceivers in Trich's circumstances of viewing.

Or:

$\left(R 1^{*}\right)$ That fruit is orange to normal perceivers in normal circumstances of viewing.

Or:

(R1) That fruit is orange to Trich in normal circumstances. 


\section{Color Experience}

Etc. By relativizing to normal perceivers, normal conditions of viewing, or objectively specified surrounds, rather than merely to Trich, Relationalists can reduce the degree of certainty that attaches to object-knowledge.

Now, $\left(R 1^{\prime}\right)$ and $\left(R 1^{*}\right)$ are not entitled to empirical certainty for reasons given earlier; examining the fruit more carefully does not give Trich certainty about how normal perceivers would experience it. (R1), however, is more promising, because it corresponds quite closely to my account of object-knowledge as far as empirical evidence goes, and Trich can adopt the same attitude to it as I earlier argued she ought to adopt with regard to OK (1). That is, when she examines it in a range of "good" circumstances of viewing, she acquires empirical certainty with regard to it.

However, the corresponding re-interpretation of object appearance knowledge (6) - the fruit looking orange - is this:

(R6) That fruit looks orange to Trich in normal circumstances and in the surround color in which it is placed.

But this is simply equivalent to (R1') on the Relationalists' account, since 'looks' is the same as 'is' for them in this context. We've lowered certainty to the desired level on being orange, but at the cost of doing the same on looking orange. The close correspondence of being a color and looking a color makes it difficult for Relationalists to differentiate the level of certainty that attaches to 'looks orange' and 'is orange'.

Difficult, but not impossible. For Cohen (2007) suggests that there actually are circumstances in which a Relationalist wants to say that something looks but is not orange. Suppose that because of some strange quirk of Trich's brain, it so happens that the smell of an orange makes whatever happens to be in Trich's hand look orange. When drinking fresh-squeezed orange juice an apple would look orange to her - and so, of course, would an orange. But a Relationalist might want to hold that this orange-look has the wrong pedigree to serve as a proper undergirding for being orange - even to 
her. In "deviant" circumstances such as these, neither the apple nor the orange is orange even though both look that way.

Because she cannot rule out this kind of possibility, Trich cannot dismiss sceptical doubt with regard to the fruit in her hand being orange - even when object-knowledge is relativized to her alone, as in (R1). Thus, her certainty regarding the color of things falls short of Cartesian demands. Nevertheless, it might be that close examination leads her to empirical certainty regarding the fruit being orange to her. On the other hand, she never has reason to doubt that it looks orange - it looks that way even under deviant circumstances. By focussing on the difference between empirical doubt and sceptical doubt, the Relationalist can thus arrive at the right balance of certainty as between 'looks' and 'is'.

This is a good result, but it is nonetheless doubtful that the Relationalist can give a proper account of the relationship of looking orange to being orange. Let me put the matter in a way that brings out the difference between their motivations and mine. I have been urging that object-knowledge and object-appearance-knowledge are about two quite different sorts of things - 'is orange' has to do with a property of an object, while 'looks orange' has to do with a perceiver's visual state with regard to that same property and that same object. The second can normally be read off the perceiver's own state, but it is evidence for the first. This accounts for the difference in Trich's epistemic entitlements with respect to these claims.

Relationalists do not recognize such a distinction - for them, the difference between 'is orange' and 'looks orange' is a contextually-defined difference about the same factual category. McLaughlin and Cohen follow the ancient Sophist, Protagoras, in matters having to do with color. One could summarize them thus:

Color-Protagoreanism In non-deviant circumstances, Man is the measure of all colors: of what is orange that it is orange, of what is not orange that it is not orange. 
Thus, they are unsympathetic to the very attempt categorically to distinguish objectknowledge from object-appearance knowledge. Further, they do not think that something's looking orange is evidence, in the normal way, for its being orange. Bracketing the weird situations of which Cohen takes note, looking orange is for them constitutive of being orange, not evidence for it.

From my perspective, this is a weakness. Intuitively, attributing a color to an external object is to attribute to it a characteristic that it possesses independently of how it might look in a particular circumstance - a characteristic that influences how it looks, but is nonetheless invariant with appearance. This is the intuition that Relationalists simply throw away.

\section{Primary Quality Theories of Color}

According to primary quality theories, color experience is no part of the essence of color. An example of a position of this kind is physicalism, according to which color is definable in the language of physics. I will not attempt a precise definition here, since it is somewhat tricky. Let us say, just so we know what kind of property we are talking about, that according to a primary quality account, color is a wavelength-related property. In the case of lights, it will be related to the relative strengths of wavelengths in the light that objects emit; in the case of transparent or translucent filters, it will be related to the proportion of each wavelength that such filters transmit as a proportion of the light that falls on them; in the case of opaque surfaces, it will be related to the proportion of light of each wavelength reflected. I shall unite these under the term "wavelength-productance," or "productance" for short (cf. Byrne and Hilbert 2003). As we shall see, the term is a place-holder: it is not definable in advance.

Now, I am sympathetic to primary quality theories, and to physicalism in particular. In my view, the colors are indeed productances, or something of this sort. (A more complicated account is needed when we include properties that modify color, such as luminescent, shiny, matte, or velveteen - see Mausfeld, this volume - but here I 


\section{Color Experience}

will consider only the colours that are described in terms of hue, saturation, and lightness.)

Here is an argument in support of primary quality theories:

Color-vision is allied, in all organisms that have it, with cognitive mechanisms that lead animals to form expectations of finding other properties that have earlier been found associated with colours. For example, if they are fed from yellow dishes and given water from green ones, then they will come to anticipate that they will find food in yellow dishes and water in green ones.

Color vision serves these animals in such contexts only because such associations are indeed predictive.

But they could not be predictive if their essence lay merely in their relationship to experience, for experience varies with circumstances of viewing.

Therefore, color vision must engage experience-independent properties.

This argument is certainly not conclusive, but it does serve as a broad motivation for a view of color that makes it a property of material objects independent of conditions of viewing.

However that might be, primary quality theories need elaboration if they are adequately to accommodate the evidentiary status of color-experience. Consider in particular the color-knowledge incorporated in CK (2) - (5) above. If no more is said about color than that it is physical wavelength-productance, it is a mystery how we could have Cartesian certainty about it (cf. Johnston 1992).

Consider, for example, (5) - the proposition that the color of the orange is closer to that of a lemon than to that of a cherry. How can Trich be certain in virtue of her own experience that the productances are related by these relations of relative 
similarity? In fact, a statistically normal human trichromat's experiences are positively misleading with respect to physical similarity. For such a color perceiver, violet is more similar to red - since both are reddish - than either is to saturated green. Yet as far as wavelengths go, green is in between violet and red. A monochrome green light would for this reason be, from the point of view of physics at any rate, more similar to monochrome red or violet lights of the same brightness than the latter are to each other. So, if colors were physical, apparent similarity would not be a reliable guide to the kind of similarity that is supposed to count for the primary quality theorist: it could be misleading that the color of an orange is more like that of a lemon than like that of a cherry, at least as far as its physical characteristics are concerned. This violates the Cartesian certainty we have been attributing to color-knowledge.

The problem is acute for missing shades. Suppose that $Y$ is a missing shade of yellow that I visually image, but, as it happens, I have never seen. According to I.C, I am entitled to Cartesian certainty about certain compositional and relational properties of $Y$. Yet, how can I possess any kind of certainty about primary qualities I have never encountered? And what about impossible shades? How can something be a primary quality if it cannot be instantiated in anything that exists in the real world?

There is also this daunting (though, as we shall see, illusory) difficulty. In physics, there are wavelengths and various properties associated with wavelength. Psychologists often note, however, that aside from particular sorts of experience, it means nothing to say that a particular wavelength is orange, or blue, or green. These categories depend on how light is experienced, and have no physical significance independently of experience. Thus, while one may agree that light of $600 \mathrm{~nm}$ is a primary quality, it is unclear how orange could be so. The essence of orange is essentially connected with the color experience of the orange-type: if light of $570 \mathrm{~nm}$ elicited this kind of experience, then it would be orange. But it is certainly possible for light of $570 \mathrm{~nm}$ to elicit this experience in creatures only a little differently constructed than we. 
Thus, it seems to follow:

(7) It is possible that light of $600 \mathrm{~nm}$ is not identical with orange.

However, by a well-established truth of modal metaphysics:

(8) (Possibly $x$ is not identical with $y$ ) implies ( $x$ is not identical with $y$ ).

So:

(9) Light of $600 \mathrm{~nm}$ is not identical with orange (or any of its determinates).

This contradicts physicalism.

The questions for the primary quality theorist, then, are first, how knowledge concerning light of $600 \mathrm{~nm}$ can possess the Cartesian certainty of color-knowledge propositions (2) - (5), and second, how experientially identified properties such as orange could be primary qualities.

\section{A Projective Semantics for Color Experience}

A. In a semantic theory, colour experiences denote colours. Just as 'cat' denotes the property that cats share, so also Trich's $\mathrm{O}$-experience denotes, or represents, a property that (some) orange things share. ${ }^{2}$ This gives color-experience a role quite different from that envisaged by secondary quality theories. It is a symbol internal to the workings of the mind, a token by which the color vision system passes to other epistemic faculties, and to the perceiver herself, the message that by means of its own data-processing, it has determined the color of this visual object to be orange.

${ }^{2}$ The $O$-experience is more determinate than orange - this is why not all orange things evoke this precise kind of experience. 
The O-experience is, I am suggesting, the semantic marker of this message semantic not in language that the perceiver herself uses (such as English or Malayalam), but in the signalling system that is used by the perceiver's cognitive apparatus.

The relationship between the $O$-experience and orange is conventional in the following sense: the experience is a marker, or label, for that color; any other experience would have served as well for the same purpose. It is important to internal functioning that this type of experience consistently be used to mark this particular color, but it is a matter of historical and genetic happenstance that that particular type of experience, rather than any other kind, is used to pass messages about this particular color property. The system could have used some other kind of experience in place of the one it in fact uses: the pairing of the $O$-experience type and orange is in this sense arbitrary. (See Matthen 2005, part IV, for a more detailed treatment of these points.)

B. A semantic theory relies on a mind-world relationship fundamentally different from those employed by the other theories we have been considering.

In some of these theories, perceivers have to know something outside the mind before they can come to know color: in Dispositionalism, perceivers have to know the starting point of a causal relation that (in normal circumstances) vectors inward from world to color-experience; in the case of Primary Quality Theories, they have to know a physical quantity. Relationalism does not fall prey to this problem, but it doesn't countenance any mind-world relation in color-experience: for this theory, for something to be colored is simply for it to be experienced this way.

Semantic theories, on the other hand, rely on an outbound relation between symbol and denotation. One grasps something of what a symbol signifies when one knows its sense, or meaning. This is what semantic theories require of color-experience. But since it is possible to grasp the sense of a symbol without being able to identify its referent or denotation, the semantic theory does not require knowledge of anything outside the mind. 
C. Some symbols refer (or denote) projectively. Consider an air-traffic controller working at a radar display. A particular aircraft on her screen is shown as a red dot. She refers to this aircraft as "the red plane". Of course, she does not mean or imply that the aircraft itself is red. She has no idea what colour it is. She is using a property of the symbol to refer to the object that it denotes. I will call this use of 'red' projective.

Some projective symbols form a structured denotational system. Think of a map with intervals between equally spaced vertical grid lines marked $A, B, C$, etc. from left to right and intervals between equally spaced horizontal lines marked 1, 2, 3, etc. from top to bottom. Let us stipulate that this is a projective system in which "Pemberton Street is in G3" means that the Pemberton Street (the external world object) is in the geographic region denoted by grid location G3 (and not that the map-representation of the street is in that grid location on the map). Here, location is projectively denoted by the denotative system formed by the symbols on the map.

Certain facts about location are implied by the structure of this projective scheme. Consider:

LK (10) C3 is a greater distance away from A1 than A3.

As stipulated in the preceding paragraph, (10) is about locations in the real space represented by the map in question - it is location knowledge, not map knowledge. Yet, we know that (10) is true, not in virtue of having measured the distance, but a priori as a result of knowing how the denotational system works (and also Pythagoras' Theorem). (10) is known a priori, but it is about the external world.

Notice that (10) is more certain than:

OK (11) Pemberton Street is in G3.

(11) requires not only knowledge of the denotational system, but also empirical information about Pemberton Street. The difference between (10) and (11) is 
reminiscent of the difference between object-knowledge proposition OK (1) and colourknowledge propositions CK (2) - (5).

D. My thesis is this:

Color-experiences constitute a structured projective denotational system.

Color experience is organized around three axes: bright-light, red-green, and blueyellow. Every color experience is a combination of values of each of these axes. These experiences are arrayed around these axes as a similarity ordering: the more similar two experiences are, the closer together they are in this system. The qualities of these experiences are projected on to what they denote. To say that something is yellow is to say that it has the color denoted by the experience we recognize as of the yellow type.

On this view, some color-knowledge is projected from our knowledge of what it is like to experience color, and from the projective topology of colour experience. The color-knowledge contained in (2) - (5) is accounted for (as I shall argue in section VIII) by innate knowledge of the denotative space of color; so also our knowledge of missing shades. For instance, CK (2) above assigns a somewhat determinate color - the color of the fruit - to an extensive region within Trich's color-similarity space - the yellowish region. Her knowledge is analogous to location-knowledge of the following sort: "C3 is contained in the regions between C2 and C4", which is about location, but known a priori as a consequence of knowing the denotational system of grid location. Trich's knowledge of CK (2) is implicit in her very perception of the fruit as orange. This perceptual state cannot be wrong about the relation stated by (2), though it might be mistaken with respect to the fruit. Propositions like (2) are "quasi-analytic": all that is needed to grasp them is knowledge of the denotative scheme - and this, I will propose, is innate. Despite their quasi-analyticity, such propositions are "substantive" - they are about relations among the colors. (cf. Williamson 2007, especially chapters 3 and 4.) 
This accounts for object color-appearance knowledge too, as in OAK (6). The content of (6) is simply that Trich's color-visual state denotes a certain color. That it denotes this color is part of what the experience means.

Object knowledge as in (1) is explained by general facts about how we use our senses to probe the world. When we look at something in different and demanding ways, our senses converge on a determination that is immune from all but skeptical doubt - i.e., from doubt that does not infect unrelated propositions about our own position or about the condition of our sensory apparatus. Our knowledge of objectcolor depends on determining occurrent facts; this is why it is inherently subject to sceptical doubt.

Our knowledge of impossible shades lies a little further afield. In any compositional language, there is always the possibility of impossible compounds 'square circle' is an example. However, it was for a long time thought impossible that a sensory system could produce such compounds - it was thought to lack the conceptual freedom that discursive thought enjoys. For this reason, it was not only thought impossible that things in the world should be both reddish and greenish, but also that one could have such an experience. But it has been shown that under certain circumstances, the visual system can be induced to produce tokens of this sort.

\section{Color Essences}

We have been discussing truths that arise from the system of sensory denotation - call these representation-relative truths. There are other propositions concerning the colors that are necessary as a consequence of what these colors are in themselves - call these color-essence truths. Representation-relative truths are necessary because of how the colors are denoted. Color-essence truths are not necessary merely under some description, but necessary without qualification. For instance, it is necessary of colors that they are related to the wavelength of electromagnetic radiation in certain ways. This is not necessity in virtue of how the colors are represented in sensation, but 
necessity in virtue of physical constitution - that is, in virtue of what color is. In fact, experiencing the colors does not afford us access to these necessary truths.

This distinction is useful to primary quality theories of color. Let's once again take the wavelength-productance view as our paradigm of this approach. According to this theory, the colour denoted by $O$ is a productance related to light of $600 \mathrm{~nm}(S)$, say. Such a productance exists independently of us. $O$ denotes $S, O$ is our sensory way of identifying it - indeed $O$ is our only way of identifying $S$ prior to physics. But the physical character of $S$ is not revealed by $O$.

Note that $O$ need not have denoted $S$. $O$ would not have stood in this relation to $S$ if our visual systems had been constructed slightly differently. If they had been so constructed that light of $590 \mathrm{~nm}$ elicited $O$, then $O$ would have denoted a productance related to light of $590 \mathrm{~nm}\left(S^{\prime}\right)$. In that case, $O$ would have been our way of identifying $S^{\prime}$.

This shows what is right and what is wrong about the argument expressed at the end of section III. On the one hand, we have knowledge of color that is dependent on the representational structure of color-experience. This knowledge includes some quasi-analytic truths. We are entitled to Cartesian certainty regarding these. However, these truths are necessary only given the relationship between color-experience and color. They are not necessary concerning colors taken in themselves. On the other hand, there are truths necessary of colors taken in themselves - but we are not entitled to certainty with regard to these in virtue of our experiences. Indeed, we may not know them at all. That color is wavelength-productance is one such truth.

Consider, then, the following propositions about color-experience and colorproperties. (In the hope that it will help the reader's intuitions, I'll place a linguistic parallel in brackets.) Given that $O$ denotes $S$ (cf. 'domestic cat' denotes felis sylvestris catus) one may say:

(12) The color that $O$ denotes is identical with $S$ (cf. domestic cat is identical with felis sylvestris catus). (Important note: Dropping the 
quotation marks around 'domestic cat' enables us to talk about the property denoted by the term. It is this property that is identical with the species, not - obviously - the term itself. Similarly, it is the color denoted by experience $O$, not $O$ itself, that is being identified with the productance.)

From (12), it follows that:

(13) The color that $O$ denotes is necessarily identical with $S$ (cf., Domestic cat is necessarily identical with felis sylvestris catus).

But (13) should not be confused with:

(14) $O$ necessarily denotes $S$ (cf. 'Domestic cat' necessarily denotes felis sylvestris catus).

(14) is false. If our color visual system had been constructed differently, $O$ would not have denoted $S$ but $S^{\prime}$. But this is not sufficient to contest (12) or (13). Though $O$ might have denoted something else, it is nonetheless true of the color denoted by $O$ that it is identical with $S$.

The proposition expressed by (13) has to do with the necessary properties of something independently of how we denote that thing. (14), on the other hand, has to do with the relationship between a meaningful symbol and something in the world. It implies nothing about this worldly thing beyond the relationship in which it stands to the symbol. (13) is about something beyond the relationship.

Primary quality theories of color are still in the running, given the semantic conception. The powerful sounding argument at the end of section III is mistaken. That argument assumes that orange must be different from the productance associated with light of $600 \mathrm{~nm}$ because the former but not the latter is essentially connected with experience. This commits a kind of de dicto/de re confusion: that something is 
necessary given how the color-vision system denotes it is confused with necessity tout court.

\section{Defining Color Similarity}

What about the color categories defined by experience? These are founded on similarity relations among the color-experiences. What guarantee do we have that these similarity relations correspond to a real similarity measure. We experience red as more similar to violet than to green, but what reason do we have for affirming any physical basis for such a similarity-grading? We have introduced a role for colorexperience in grasping color-properties. But shouldn't a semantic theorist eschew appeal to experienced similarity to ground real similarity? Since experience is a symbol for color, it is a bit like saying that cats are similar to rats because the word 'cat' is similar to the word 'rat'. The similarity of color-experiences seems an unimportant and inconsequential basis for a real-world similarity relation that is meant to be cognitively important.

One position that one might take here is that nature has chosen to reveal the similarity of colors to us in color-experience. Color-experiences are experiences of color; color-similarity experience is similarly experience of their similarity. Experiencing two colors as similar is not a matter of having similar experiences of them. In other words, color-similarity experience does not consist merely in transferring an incidental similarity relation from experiences over into the realm of colors. It is rather an experience that tracks similarity. This is a more meaty position than the one outlined in the preceding paragraph, namely that the similarity relation revealed by color experience is nothing more than a projection from a similarity relation on colorexperiences..

As it stands, however, this more meaty account is unsatisfying. The claim is that color-experience tracks an independently valid similarity relation among physical colors. What similarity relation is that? We do not want to end up saying: $x$ and $y$ are experienced as similar whenever they stand in physical relation $P$, therefore, $P$ is the 


\section{Color Experience}

relation we experience as color-similarity. Such a theory does not advance beyond the account outlined at the start of this section. What is needed is some independently motivated account - something that could make us understand what real relation experiential similarity tracks.

Purely physical accounts do not suffice for this purpose. In section III, we encountered one important reason for thinking that wavelength based and other physical accounts are inadequate: color experience distorts the physical spectrum, making violets closer to red than to green. Another reason in the phenomenon known as metamerism: colors that are physically quite different from one another can look the same because they have the same effect on the color receptors in the retina.

I would like to propose an alternative account of the basis of sensory similarity relations. They are the basis of conditioning. Let's suppose that feature $F$ is associated with feature $G$. Given certain other conditions about the naturalness of $F$ and $G$, an animal that has been exposed to this association will act in the presence of $G$ as if $F$ were presented. In other words, the subjective probability that the animal attaches to $F$ rises when it senses $G$ - its response is conditioned by the association of $G$ with $F$. It is important to note that the conditioning function - the function that determines how incoming data modifies responses - is itself an unconditioned response. That the animal adopts a conditioned response to $F$ when it finds $F$ associated with $G$ is itself an unconditioned, or innate, response to the association. The perception of $F$ together with $G$ results in a modification of the animal's epistemic state.

Now, what happens when $G^{\prime}$, which is slightly different from $G$, is presented? The answer is this: the rise in the subjective probability in $F$ will depend on how similar $G^{\prime}$ is to $G$. The more similar it is, the greater the probability that the response conditioned on $F$ will be triggered. In fact, this is how similarity space is measured in animals: $G$ is similar to $G^{\prime}$ to the degree that conditioned responses to $G$ pass over to $G^{\prime}$. I shall take this as a definition of similarity - call it similarity-for-conditioning. Thus: 
Similarity-for-conditioning For any animal, $x, G$ is similar to $G^{\prime}$ to the degree that $x$ 's unconditioned responses to $G$ would overlap, over a long series of trials, with its unconditioned responses to $G^{\prime}$.

Here, I should emphasize again that forming a conditioned response - that is, the act of forming such a response - is itself an unconditioned response. The latter unconditioned response is dynamic: it is a change in epistemic state (i.e., a change in subjective probabilities) consequent to a perceptual state.

This conception of color-similarity is based on the unconditioned propensity to form conditioned associations. I am assuming that experienced similarity is innately so set up as to track similarity-for-conditioning (not physical similarity). This is not analytically true, or even a priori true. It could have happened that experienced similarity had contours quite different from those of similarity-for-conditioning. Nevertheless, it is empirically quite plausible that experienced similarity closely follows similarity-for-conditioning. For in the first place, it does seem that humans unconditionally form associations based on conscious sensory experience. For example, if an experimenter delivers an air-puff to your eye just after activating a flashing red LED, a subsequent activation of the LED will trigger an involuntary blink even if no airpuff is forthcoming - here, your experience of the LED is connected by association with the air-puff. Moreover, as I said earlier, this is what students of comparative vision assume when they measure the similarity relations of animals that cannot speak. The assumption could in principle be tested by figuring out whether the two measures of similarity diverge for humans - as far as I know, this has never been done.

The proposal that color-similarity is similarity-for-conditioning may strike some as not very different from the weaker proposal mentioned at the beginning of this section, namely that it is merely experienced similarity. Indeed, I have just reinforced this impression by suggesting that similarity-for-conditioning probably coincides with experienced similarity. Is experienced similarity merely subjective, then, in the way that experience itself is regarded as merely subjective? 
I do not think so. My proposal explicates experienced similarity. To say merely that two colors are similar to one another when they are so experienced gives us no idea what the content of the experience is. As such, it leaves it open that colorsimilarity is an isolated phenomenon that has nothing at all to do with any extraexperiential realm. The proposal that experienced similarity is similarity-forconditioning does better than this. It makes clear what the stakes are in experiential similarity. Two things are experienced as similar to the degree that certain learned expectations regarding the one spread to the other. So experiential similarity represents a certain color-associated clustering of properties in external things. To experience two things as similar is to experience them as sharing other properties as well. Similarity-for-conditioning is a dynamic interpretation of similarity: it links similarity of color to certain processes within the organism.

\section{Pluralistic Realism}

One important corollary of identifying color-similarity relations with similarity-forconditioning is that it opens up the possibility that these relations could vary from species to species. Animals of different species live in different environments, and undertake different activities in order to live in these environments. They also vary in how they receive color information: they have different numbers of color-sensitive receptors, which may be tuned differently; some have filters; in invertebrates, the eye itself is differently structured than in vertebrates. These differences make for significant differences in the domain and contour of color-similarity relations among animals (Matthen 1999). Not only do they receive different information from the environment (because their receptors are different), but they also process this information differently in order to best serve the data-processing needs of their styles of living. Further, their unconditioned responses to sensory data are different - both in terms of innate behavioural response, and in terms of how conditioning works.

The variability of color across species has an important consequence. One cannot say a priori what color is - it is different things to different kinds of animal. Of 
course, it involves discrimination based on the wavelength of light. But beyond this there are important differences. For example, information about the polarization of light is visible to birds and bees "in color"; it is more or less invisible to humans. How do secondary quality theories handle this? According to these theories, color is the property of normally causing certain sensations? Which sensations? There is no guarantee that polarization sensations in birds is the same as those (if any) in bees, and none that these sensations correspond to those by which humans perceive color. So this approach to defining color runs into difficulties with identifying bird-color. A properly comparative approach to color vision should not face such difficulties.

Similarity-for-conditioning defines color-similarity from the organism's point of view. Let's call it primary similarity. It is possible to investigate the physical correlates of primary similarity. For instance, psychophysicists are interested also in discovering the physical similarities that hold between objects that are united by primary similarity. In what physical respect do two things have to resemble each other when they elicit the same conditioning responses? In what physical respect must they differ (despite other similarities) when they elicit different conditioning responses? The answers to these questions will determine the physical denotation of the internal representation of color. I'll call this secondary color similarity. (cf. Matthen 2005, 232-234). It is by the psychophysical investigation of secondary similarity that we arrive at a specification of "productance".

The relationship between primary and secondary similarity is somewhat analogous to that between Frege's sense and denotation: primary similarity describes color similarity from the perspective of the organism; secondary content identifies the real-world realization of the organism's similarity relations. The properties and relations denoted by color vision exist independently of the organism - they are objective, and in this way, the account I have offered is a primary quality account. On the other hand, our grasp of these primary qualities is through similarity relations realized by our mental dispositions. In fact, this is our primary grasp of such qualities - even though we could 
in principle come to know of these qualities in a physics class, it is nonetheless true that physicists identify colors by means of human experiences - the problem raised at the end of section III is solved by noting that orange is simply that physical variable that a particular type of color-sensation denotes. That sensations are in this way epistemically indispensible for identifying the colors accounts for why color has been thought to be response-dependent, or a secondary quality.

My position is realistic. But on my position, primary color similarity varies across species, as does secondary similarity. In virtue of this variation, the position is pluralistic - it allows that color is realized in different ways in different organisms. The position can be entitled Pluralistic Realism (cf. Matthen 1999, 2005).

\section{Similarity Spaces and Color Knowledge}

A similarity space is a graphical representation of comparative similarity relations on some domain. In the case of color, the points in this space are colors - or productances. The distance between two points in this space is inversely proportional to the degree of similarity between them. Sometimes, a similarity space is represented as a geometrical solid. The advantage of such a representation is that certain lines or axes of such a solid are thought to isolate some fundamental parameter of variation in color. Color has often been represented as a double cone (cf. Churchland, Kuehni, this volume), with the polar axis representing lightness/darkness, radial distance from this axis standing for saturation, and points on the equator standing for saturated hues. Most color scientists take this to be at best an approximation (or perhaps worse: Mausfeld, McLeod, Niederée, this volume). Nothing in my presentation depends on a strong interpretation of the color solid.

My thesis is first of all that colour vision assigns each object to a place in this space. Each such place corresponds to an experience, which denotes a productance (or class of productances). Each such place also links to conditioning: when a place in the color similarity space is found co-instantiated with some other sensory feature, a 
conditioning link is established: any occurrence of either of these will increase the subjective probability of the other.

The propositions that we know by color-experience have to do with relations among colors in this similarity space. For example, consider again:

CK (2) The color presented in $O$ is yellowish.

This tells us that the productance in question is contained in that part of color similarity space that is denoted by experiences of the yellowish kind. The containment here is revealed in a special way:

Color $X$ contains $Y$ if $Y$ is one pole of a hue-dimension (blue or yellow; red or green) or $Y$ is light or dark, and $X$ is located in the part of color similarity space denoted by $Y$.

This use of 'contains' is no doubt encouraged and influenced by some quality of colourexperience - for example, the experience of turquoise has discernible elements of bluishness and greenishness. The important thing to understand here is that we are not compelled to treat containment as either a primary relationship that exists independently of color-experience or a secondary one that simply transfers to colors from experience as the result of some cognitive illusion. It can be true of colors in virtue of a cognitively important relation between experience and reality.

Now to the evidentiary status of color experience. I suggested at the outset that color vision maps distal objects into an innate similarity space. Famously, Noam Chomsky proposed that the deep syntactic structure of language is innate. A few years after human infants have been exposed to some language, they have a nearly comprehensive grasp of the grammatical principles of this language. They understand sentences they have never heard before; they understand how to transform sentences in interrogative form into sentences in indicative form; they know when a verb they have never heard before agrees or fails to agree with its subject - and so on. 
The view that I am proposing is that, analogously, we possess innate knowledge of the similarity space of color. This is implied by the idea that similarity-for-conditioning is unconditioned, and hence innate. The innateness of color space accounts for our knowledge of the "quasi-analytic" propositions, (4) - (7). Such knowledge emerges in development, and a certain amount of experience of the right kind is needed for that emergence. Nevertheless, the relations of relevance that exist between the kind of experience needed for the emergence of innate knowledge and that knowledge are not sufficient for calling the former evidence for the latter - the stimulus is insufficient to the kind of knowledge that emerges from it. The requisite color experience is merely a causal condition for the expression of something "in the genes".

This view particularly well accounts for our knowledge of missing and impossible shades. Consider Trich's knowledge of a missing or impossible shade, or of similarity relations such as that mentioned in (7). She knows these by knowing the similarity space of color and its component structure. Knowing how these components combine makes it possible for her to combine them in a way that produces images of colors she has never before experienced; decomposing them allows her to know the structure of impossible shades though they can never be instantiated in the world. Since a perceptual grasp of the similarity space of color is a precondition of her sensing the color of the fruit in the first place, it is not, as it were, an additional burden for the account.

Finally, on this point, consider the variability of color perception from individual to individual within a single species. It commonly occurs that one person sees a particular colour chip $B$ as saturated blue with no admixture of red or green (i.e., as "uniquely blue"), while another sees it as a somewhat greenish blue. (This, by the way, is a reason why it is wrong to suppose that things elicit from all other perceivers the same sensations as they do from her.) Such a difference is often accompanied by agreement with respect to colour matching - the two persons may mostly agree when asked whether two chips are of the same colour, and this may be so across the whole 


\section{Color Experience}

range of colours. Asked whether $B$ is the same or different from other chips, they mostly agree - though they continue to disagree about whether $B$ is uniquely blue. The question is: What is the content of this perceptual difference? What are two people disagreeing about when one sees $B$ as uniquely blue, and the other sees it as possessing an admixture of green?

On the secondary quality view, the disagreement is about the external world. In one perceiver, $B$ is disposed to create a sensation of unique blue, and hence has the color-disposition associated with this sensation; relative to the other, it is differently disposed. Thus, it appears a different color to these different perceivers. Given that such differences are possible, neither can possess certainty with regard to whether anything produces a particular sensation in perceivers other than themselves. Thus, neither perceiver can be certain of the color of the fruit. And this uncertainty does not go away with careful examination. Examining a fruit closely, turning it over, taking it over to the window, putting on one's glasses: none of these strategies will elevate one's level of confidence concerning the fruit's color, at least not as far as what sensation it evokes in other perceivers. On a primary quality account similarly, there is real disagreement: according to one perceiver a thing of $B^{\prime}$ s productance is unique blue, according to the other it is not (cf. Tye 2006, Byrne and Hilbert 2007). Yet, it is hard to say what exactly the disagreement is about. What is it for a productance to be unique blue in itself, i.e., independently of anybody's perception of it?

On a semantic theory, the problem goes away. In one perceiver, the color of the fruit is denoted by the experience that occupies one position in color-similarity space; in the other it is denoted by the experience that occupies another. This is not a difference in what color is denoted, i.e., what productance. Rather, it is a difference in how it is denoted - by what kind of sensation. Though I have been talking about inter-species differences, this kind of variation can occur even among individuals of the same species. Certain transformations of internal representational schemes make no difference to the information that is contained in them - and thus they do not imply a difference of the 
information carried by experiences. For unknown neurological reasons, such insignificant transformations do occur quite frequently. Thus, it sometimes happens that one representational scheme has a blue-yellow hue dimension that is slightly rotated relative to another. Consequently, an object that falls directly on this axis in one lies slightly off it in the other. In the on-axis case, the chip is seen as uniquely (or truly) blue. When the chip falls off-axis, it is seen as not uniquely blue. Though the experiences are different, they denote the same productance.

Conclusion The view that I have been propounding is that the relationship between color experience and color is semantic. Such a view is quite natural if one takes perceptual states to be either true or false. For since it is propositions that are either true or false, the view that perceptual states have intentional content implies that they are directed in some way towards propositions. Now, propositions contain properties as constituents. Hence, it is natural to suppose that perceptual states are directed in some way towards properties. This is the consequence of the view that I have been developing: color perceptual states denote color properties. Since the view that perceptual states are either true or false is pretty widely held, it is somewhat surprising that the semantic view of color-experience has not been more thoroughly explored. 


\section{REFERENCES}

Byrne, Alex, and David R. Hilbert. 2003. Color Realism and Color Science. Behavioral and Brain Sciences 26:3-21.

Byrne, Alex, and David R. Hilbert. 2007. Truest blue. Analysis 67, no. 293:87-92.

Churchland, Paul. On the Reality (And Diversity) of Objective Colors: How Color Qualia Space is a Map of Reflectance Profile Space. In this volume.

Cohen, Jonathan. 2004. Color Properties and Color Ascriptions: A Relationalist Manifesto. Philosophical Review 113, no. 4:451-504.

Cohen, Jonathan. This volume.

Cohen, Jonathan. 2007. The Relationalist's Guide to Errors About Color Perception. Noûs 41: 335-353.

Crane, Hewitt D., and Piantanida, Thomas P. 1983. On Seeing Reddish Green and Yellowish Blue. Science New Series 221 , no. 4615:1078-1080.

Hellie, Benj. 2005. Noise and Perceptual Indiscriminability. Mind: A Quarterly Review of Philosophy 114, no. 455:481-508.

Johnston, Mark. 1992. How to Speak of the Colors. Philosophical Studies: An International Journal for Philosophy in the Analytic Tradition 68, no. 3:221-263.

MacLeod, Don. Into the Neural Maze. In this volume.

Matthen, Mohan. 1999. The Disunity of Color. Philosophical Review 108:47-84.

Matthen, Mohan. 2005. Seeing, Doing, and Knowing: A Philosophical Theory of SensePerception. Oxford: Clarendon Press.

Mausfeld, Rainer. Colour within an internalist framework: The role of 'colour' in the structure of the perceptual system. In this volume. 


\section{Color Experience}

McLaughlin, Brian P. 2003. Colour, Consciousness, and Colour Consciousness. In Consciousness: New Philosophical Perspectives, Smith, Quentin (ed).

Niederée, Reinhard. More Than Three Dimensions: What Continuity Considerations Can Tell Us About Perceived Color. In this volume.

Tye, Michael. 2006. The puzzle of true blue. Analysis 66, no. 291:173-178.

Williamson, Timothy. 2007. The Philosophy of Philosophy. Oxford: Blackwell. 